\title{
Composite Si/PS membrane pressure sensors with micro and macro-porous silicon
}

\author{
L SUJATHA and ENAKSHI BHATTACHARYA
}

Microelectronics and MEMS Laboratory, Electrical Engineering Department, Indian Institute of Technology Madras, Chennai 600036

e-mail: enakshi@ee.iitm.ac.in

\begin{abstract}
Porous Silicon (PS) is a versatile material with many unique features making it viable in the field of Microelectromechanical Systems (MEMS). In this paper, we discuss the optimization of formation parameters of micro and macro PS with different porosity and thickness for use in pressure sensors. The optimized material is used in the fabrication of composite $\mathrm{Si} / \mathrm{PS}$ membranes in piezo-resistive pressure sensors and tested. Pressure sensors with composite membranes have higher sensitivity than those with single crystalline silicon membrane with the sensitivity increasing as the porosity increases. For the same porosity and thickness of the PS layer, Si/micro PS membranes exhibit higher sensitivity than Si/macro PS ones. The offset voltage in these sensors is found to be high and can be due to the stress induced in the membrane during PS formation. Offset voltage and stress values are found to be higher in composite membranes with micro PS as compared to macro PS.
\end{abstract}

Keywords. Porous silicon; MEMS; pressure sensor; porosity.

\section{Introduction}

Understanding the formation theory, morphology, chemical and mechanical properties of PS are essential for its successful use in new MEMS applications. MEMS pressure sensors made of silicon are commercially available (Senturia 2001). They essentially use a thin silicon membrane that deforms under pressure. The deformation can be measured capacitively (Tai-Ran-Hsu 2002) or by placing piezo-resistors on the membrane (Stephen Beeby et al 2004) and noting the change in resistance due to deformation. Bhat et al (2004) have reported on bulk micromachined pressure sensors fabricated with single crystalline silicon membrane and polysilicon piezo-resistors on oxide layer that exhibit good sensitivity and temperature tolerance. The sensitivity of the sensor depends on the thickness of the membrane. For measurement of low pressures with standard pressure sensors, the silicon membrane has to be very thin. Use of very thin membranes to get higher deformation leads to difficulty in handling and also introduces ballooning effect (Timoshenko \& Woinowsky 1959). The devices show non-linearity when their deformation is comparable to the membrane thickness. Using a Si/PS composite membrane where the silicon membrane is converted into PS by electrochemical etching to a certain depth can be useful for measuring low pressures. Since PS has a lower 
Young's Modulus, the deformation under pressure is more and the strain is also greater as compared to that of the silicon membrane. Hence the sensitivity of the composite membrane can be increased without making the membrane too thin. We have earlier reported on pressure sensors with composite Si/micro PS membranes (Sujatha \& Enakshi Bhattacharya 2007). In this paper, we make composite membrane pressure sensors with macro PS and compare the performance with that of micro PS. PS as pressure sensing material due its higher piezoresistive coefficient has been reported by Pramanik et al (2005). We exploit the mechanical properties of PS with the composite membrane pressure sensors.

\section{Formation and optimization of PS for pressure sensor}

PS is formed by electrochemical etching of Silicon in HF based electrolyte. The substrates were $p$-type (100) silicon with resistivity of $1-1 \mathrm{ohm}-\mathrm{cm}$. The formation parameters such as current density, anodisation time and electrolyte composition were varied to modify the porosity and thickness of the PS layer. The porosity and thickness of PS were measured by gravimetric method as given by Ossicini et al (2004). The optimization of PS thickness is based on the total membrane thickness which is $16 \mu \mathrm{m}$. We found the formation conditions to convert $6 \mu \mathrm{m}$ thickness of silicon membrane into PS with different porosity. Earlier reported results show that the sensitivity of the pressure sensors increases with the increases in the thickness of the PS layer (Sujatha \& Enakshi Bhattacharya 2007).

\subsection{Micro PS formation and optimization}

Micro PS is defined as porous silicon with the average pore diameter less than $10 \mathrm{~nm}$ (Foll et al 2002). It can be formed on $p$-type substrate with aqueous HF-based electrolyte. The formation mechanism was studied by Smith \& Collins (1992). In our work we have used HF and IPA as the electrolyte. We measured the average pore diameter in the range of $5 \mathrm{~nm}$. Figure 1 shows the SEM image of micro PS formed with the formation conditions as HF:IPA = 1:1; Current density $=10 \mathrm{~mA} / \mathrm{cm}^{2}$ and the anodisation time $=10$ minutes.

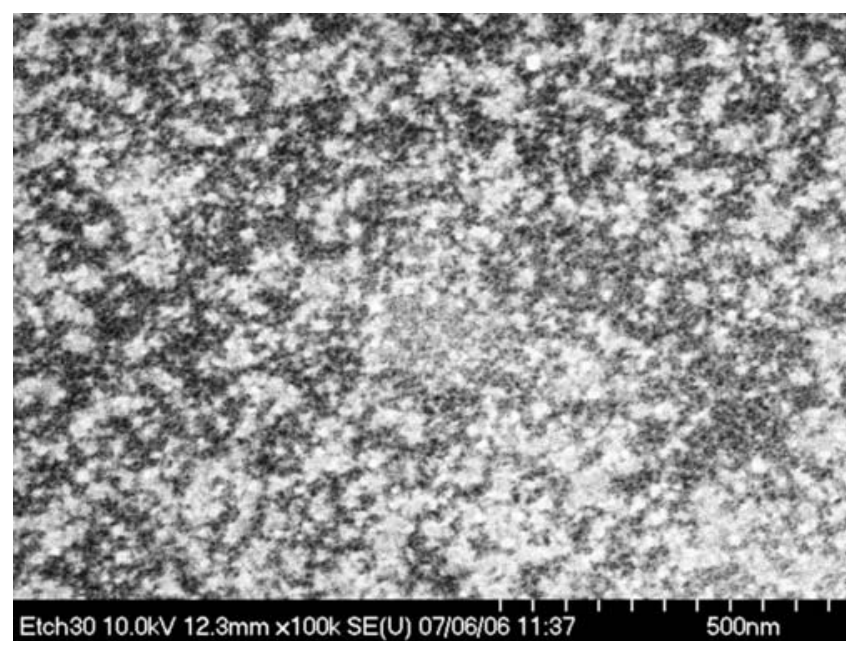

Figure 1. SEM image of microPS formed on a $p$-type substrate. 
Table 1. Micro PS formation conditions.

\begin{tabular}{ccccc}
\hline HF $(\%)$ & $\begin{array}{c}J \\
\left(\mathrm{~mA} / \mathrm{cm}^{2}\right)\end{array}$ & $\begin{array}{c}\text { Time } \\
(\mathrm{min} .)\end{array}$ & $\begin{array}{c}\text { Porosity } \\
(\%)\end{array}$ & $\begin{array}{c}\text { Thickness } \\
(\mu \mathrm{m})\end{array}$ \\
\hline 80 & $7 \cdot 5$ & 12 & 50 & 6 \\
50 & 5 & 20 & 70 & 6 \\
33 & 12 & 10 & 90 & 6 \\
\hline
\end{tabular}

The optimised formation parameters to get PS layers with $6 \mu \mathrm{m}$ thickness and different porosity for a composite membrane are given in table 1.

\subsection{Macro PS formation and optimization}

Usually macro PS is formed using $n$-type Si substrates with aqueous electrolyte. Since the electrochemical etching requires the presence of holes, the $n$-type substrates have to be illuminated from the back for the supply of holes by photogeneration (Lehmann 1993). This arrangement also requires the IR light from the source to be filtered out to avoid the heating of the substrate. This makes the set-up complicated.

Ponamarev \& Levy Clement (2000) have reported on using organic electrolytes to form macro PS on $P$-substrates with properties rivaling those of perfect $n$-type macro PS. Since the substrate is $p$-type, there is no need for back illumination. Following Vyatkin et al (2002) we have made macro PS with HF and Dimethyl Formamide. Figure 2 shows surface profiler view of micro PS and macro PS made on the same $p$-type wafer. Pore formation in the micro PS is random whereas in the macro PS it is more regular with pore diameters in the order of few micrometers. Table 2 shows the formation parameters required for specific porosity and thickness of macro PS formation.

\section{Young's modulus of PS}

Bellet et al (1996) reported that the Young's modulus of PS is two orders less than the Young's modulus of single crystalline silicon. For isotropic PS, Populaire et al (2003) gave the expression for Young's modulus of PS $\left(E_{p s}\right)$ as

$$
E_{p s}=E_{S i}(1-P)^{3}
$$

where $P$ is the porosity, $E_{S i}$ is the Young's modulus of silicon.

Table 2. Macro PS formation conditions.

\begin{tabular}{ccccc}
\hline HF $(\%)$ & $\begin{array}{c}J \\
\left(\mathrm{~mA} / \mathrm{cm}^{2}\right)\end{array}$ & $\begin{array}{c}\text { Time } \\
(\mathrm{min} .)\end{array}$ & $\begin{array}{c}\text { Porosity } \\
(\%)\end{array}$ & $\begin{array}{c}\text { Thickness } \\
(\mu \mathrm{m})\end{array}$ \\
\hline 80 & 8.3 & 9 & 50 & 6 \\
60 & 5 & 10 & 70 & 6 \\
50 & 8.3 & 15 & 90 & 6 \\
\hline
\end{tabular}




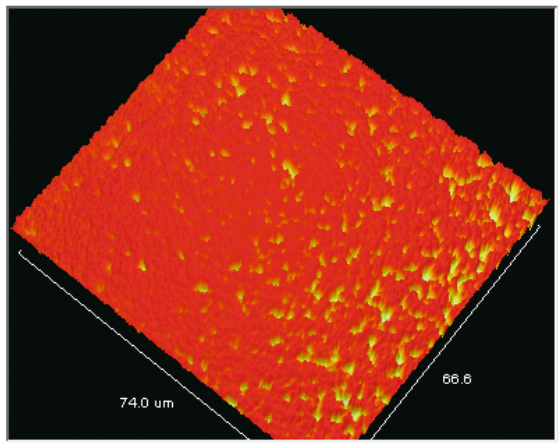

(a)

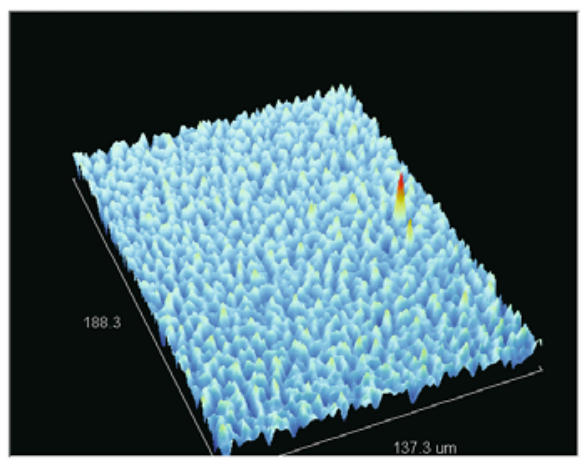

(b)

Figure 2. Profiler view of PS. (a) Micro PS; (b) Macro PS.

The Young's modulus of micro PS formed on $p$-substrate $(1-10 \mathrm{ohm}-\mathrm{cm})$ with aqueous electrolyte with $50 \%$ and $70 \%$ porosity were measured to be $22.11 \mathrm{GPa}$ and $5.51 \mathrm{GPa}$ respectively by nano-indentation. The measured values matched well with the values of $23.75 \mathrm{GPa}$ and $5.13 \mathrm{GPa}$ calculated from equation 1 . The measurement could not be done for the $90 \%$ porous sample due to high surface roughness. The Young's modulus of $90 \%$ porous PS was calculated as $0.19 \mathrm{GPa}$ using the equation 1 . These values are used in simulations of the pressure sensors to estimate the sensitivity (Sujatha \& Enakshi Bhattacharya 2007). We also used equation (1) to calculate Young's modulus of macro-PS though it is a less isotropic material. Measured values of Young's modulus were found to be lower than those calculated for macro PS.

\section{Fabrication and testing of $\mathrm{Si} / \mathrm{PS}$ composite membrane for pressure sensor}

A double side polished $p$-type $1-10 \mathrm{ohm}-\mathrm{cm}$ silicon wafer of $200 \mu \mathrm{m}$ thickness was used to fabricate a membrane of thickness $16 \mu \mathrm{m}$ by $\mathrm{KOH}$ etching from the back with oxide as mask. RCA cleaning was done and aluminium was deposited on the top plain side of the wafer by thermal evaporation to get the contact for PS formation. The wafer was scribed into $2 \times 2 \mathrm{~cm}$ to fit into the cell. PS was formed with the formation parameters optimized to obtain different porosity with same thickness. After formation of PS the back metal is removed and then oxidized to provide isolation for the sensor. Oxidation was carried out in 2 steps - first at low temperature of $450^{\circ} \mathrm{C}$ (dry) for 30 minutes and then at high temperature of $800^{\circ} \mathrm{C}$ (dry-wet-dry) for $1.5 \mathrm{~h}$. This two-step process avoids the collapsing of the Si filaments in the PS at high temperature. Blue photoluminescence (PL) under UV illumination was observed from the oxidized PS confirming the porous nature. Realizing polysilicon piezo-resistors and metal contacts as mentioned in our earlier report (Sujatha \& Enakshi Bhattacharya 2007).

To compare the behaviour of composite membranes with micro PS and macro PS the experiments were done with same membrane thickness of $16 \mu \mathrm{m}$. By controlling the formation parameters both types of PS for porosities of 50, 70 and $90 \%$ with PS layer thickness of $6 \mu \mathrm{m}$ were made on the silicon membranes. After the complete fabrication of pressure sensor as mentioned above, the pore morphology at the back of the membrane was observed on the surface profiler before measuring the sensitivity which confirmed the chemical treatments in our fabrication process were not affecting the pore structure. The PS surface has a tendency 


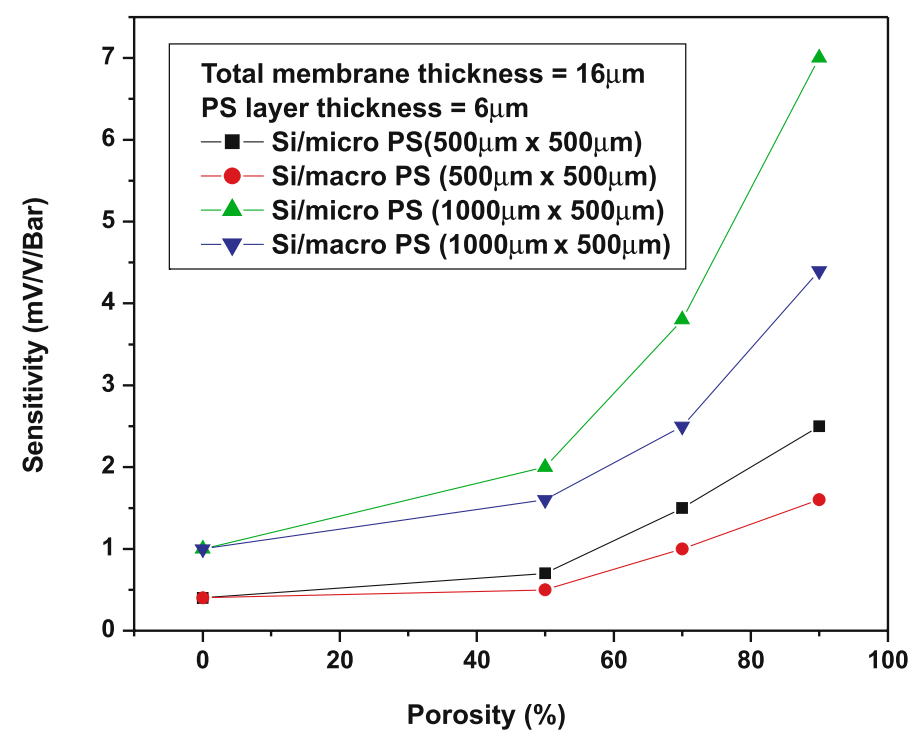

Figure 3. Sensitivity plot of Si/PS composite membrane with micro PS and macro PS.

to adsorb moisture from atmosphere. To avoid this, the samples were given a short duration bake $\left(10 \mathrm{~min}\right.$. at $\left.120^{\circ} \mathrm{C}\right)$ before the sensitivity measurement. An input voltage of $1 \mathrm{~V}$ DC was applied to one of the diagonal arms of the Wheatstone bridge formed by the polysilicon piezoresistors. The output voltage was measured at the other diagonal arm. The output voltage at 0 bar pressure gives the offset-voltage and the difference of output voltage at 0 bar and 1 bar gives sensitivity in $\mathrm{mV} / \mathrm{V} / \mathrm{Bar}$.

\section{Results and discussion}

\subsection{Sensitivity}

Figure 3 shows the sensitivity variation with porosity on both types of membranes of size $500 \times 500 \mu \mathrm{m}$ and $1000 \times 500 \mu \mathrm{m}$, zero porosity corresponds to the silicon membrane. The results show that the sensitivity increases with change in size/shape of the membrane from $500 \times 500 \mu \mathrm{m}$ (square) to $1000 \times 500 \mu \mathrm{m}$ (rectangular). This is due to geometry effect and is also seen in the silicon membranes but is enhanced for the $\mathrm{Si} / \mathrm{PS}$ composite membranes. The composite membranes show higher sensitivity as compared to silicon membranes and the improvement is higher for composite membranes with micro PS as compared to macro PS. The reason for the higher factor of increase in sensitivity obtained in $\mathrm{Si} /$ micro PS composite membrane is due to its very low Young's modulus. The Young's modulus of micro PS formed on $p$-substrate is an order lower compared to Young's modulus of meso PS formed for the same porosity as measured by nano-indentation by Bellet et al (1996). Also the random distribution of pores in micro PS may also be the reason for obtaining very high sensitivity.

\subsection{Linearity}

The composite membranes show non-linearity and also irreversible deformation at higher pressures (Sujatha \& Enakshi Bhattacharya 2007). In the present case, the devices were tested 


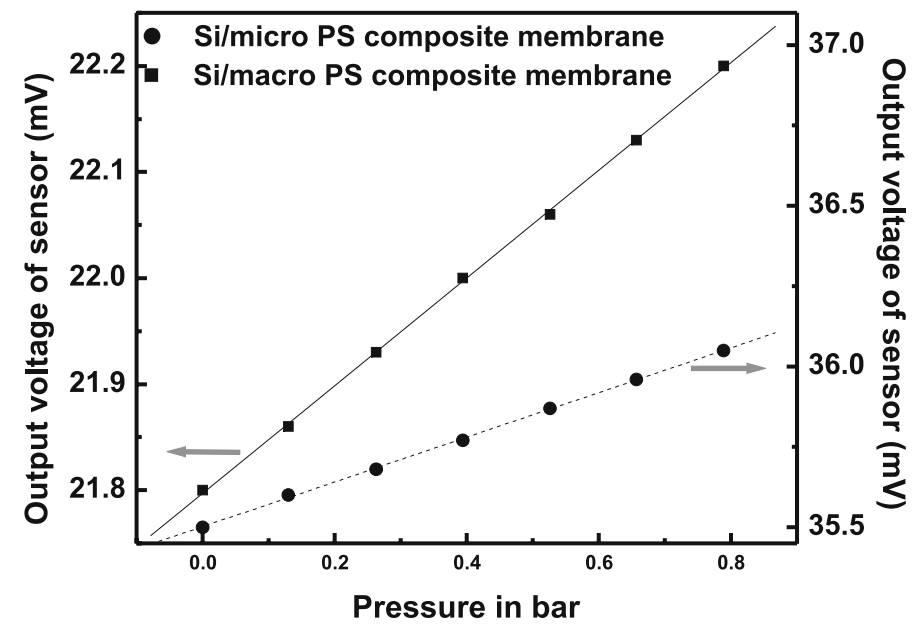

Figure 4. Linear behaviours of Si/micro PS and Si/macro PS composite membranes with $50 \%$ porosity.

at pressures less than 1 bar with a pump and manometer set-up to measure sensitivity for pressures from 0-600 $\mathrm{mm}$ of $\mathrm{Hg}$. Figure 4 shows the output voltage vs. pressure plots obtained for composite membrane with (a) micro PS (50\% porosity) and (b) macro PS (50\% porosity) on a membrane size of $500 \times 500 \mu \mathrm{m}$ and show linear behaviour in this range.

\subsection{Offset voltage}

Figure 5 shows the variation of offset voltage with porosity on $500 \times 500 \mu \mathrm{m}$ silicon and composite membranes with micro and macro PS. While the offset voltage values for silicon

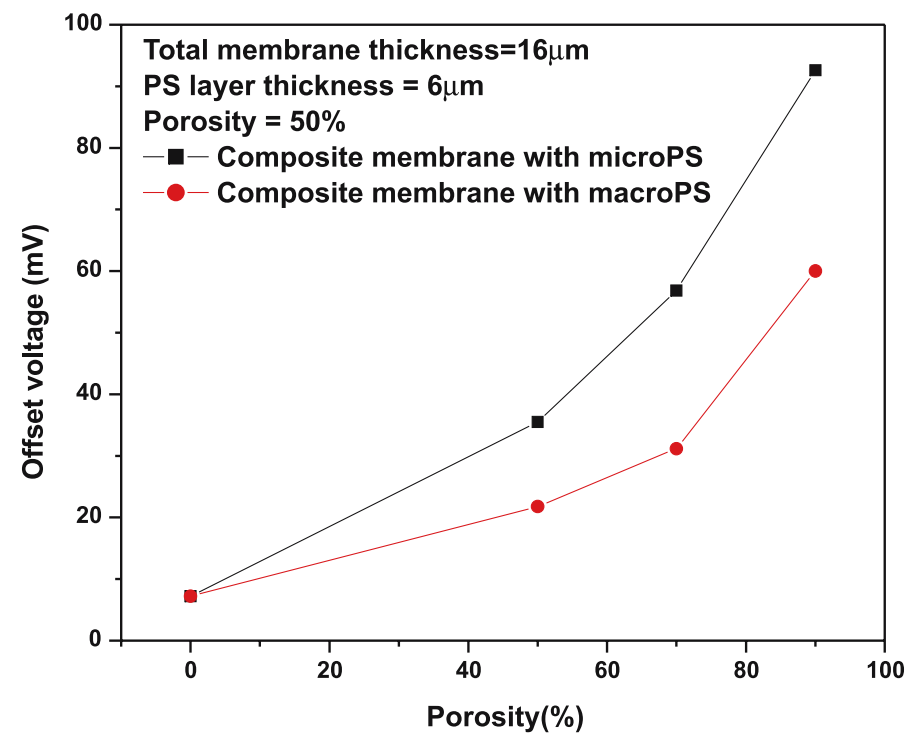

Figure 5. Increase of offset voltage with porosity in composite membranes. 
membranes are always less than $10 \mathrm{mV}$, the composite membranes show higher offset voltage that increases with porosity.

The offset voltages are much higher for micro PS-based composite membranes compared to macro PS-based composite membranes. The formation of PS by electrochemical etching develops compressive stress as reported by Manotas et al (2001). The larger offset voltage could be due to the compressive stress developed by PS formation on the membrane and may be higher for the micro PS formation compared to macro PS formation for the same porosity and thickness. The deformation in the membranes was measured using a surface profiler and the stress was estimated for composite membranes with micro PS and macro PS. The stress increases with porosity and was indeed found to be greater in micro PS membranes. More studies should be carried out on as formed PS membrane and the stress variation after each processing step e.g. oxidation, LPCVD, diffusion and annealing and steps have to be taken to reduce the stress and hence the offset voltage.

\section{Conclusion}

We have fabricated pressure sensors with composite Si/PS membranes. The sensitivity of composite membranes is found to be higher than single crystalline silicon membrane and increases with the porosity of the PS layer. It was found that for the same porosity and thickness, the composite membranes with micro PS show higher sensitivity than those with macro PS. The composite membranes were found to exhibit a linear response at pressures below 1 bar. Formation of PS results in stress and deformation in the membranes giving higher offset voltages for the pressure sensors with composite membranes than with silicon membranes. This effect is greater in $\mathrm{Si} /$ micro PS than in Si/macro PS membranes. Though the composite membranes show non-linearity at higher pressures, it is a viable option for measurement of low pressures.

We thank Dr Sara Stolyarova of Technion, Israel for the SEM and Prof. U Ramamurthy of Indian Institute of Science, Bangalore for the nanoindentation measurements. We acknowledge helpful discussions with Prof K N Bhat. This work was supported by the Asian Office of Aerospace Research and Development (AOARD), Air Force of Scientific Research, USA.

\section{References}

Bellet D, Lamagnere P, Vincent A, Brechet Y 1996 Nanoindentation investigation of the Young's modulus of porous silicon. J. Appl. Phys. 80(7): 3772-3776

Bhat K N, Bhattacharya E, Das Gupta A, Das Gupta N, Rao P R S, VinothKumar V, Sivakumar K, Manjula S R, Madhavi S P, Daniel R J 2004 Silicon micromachining and SOI technology for pressure sensors. Proc. of Int. Syposium on Smart Materials and Systems, Chennai, December 15-17, 41-51

Foll H, Christophersen M, Carstensen J, Hasse G 2002 Formation and application of porous silicon. Mater. Sci. and Eng. R39: 93-141

French P J 2002 Polysilicon: A versatile material for Microsystems. Sensors and Actuators A99: 3-12

Lehmann V 1993 The physics of Macropore formation in low doped $n$-type silicon. J. Electrochem. Soc. 140(10): 2836-2843 
Manotas, Agullo Rueda F, Moreno J D, Ben Hander F, Martinez J M Duart 2001 Lattice - mismatch induced-stress in porous silicon films. Thin solid films 401: 306-309

Ossicini S, Pavesi L, Priolo F 2004 Light emitting silicon for microphotonics $1^{\text {st }}$ Ed., (Berlin: Springer Publications)

Ponamarev E A, Levy Clement C 2000 Macropore formation on p-type silicon. J. Porous Materials (7): $51-56$

Populaire Ch, Remaki B, Lysenko V, Barbier D, Atrmann H Pannek T 2003 On mechanical properties of nanostructured meso-porous silicon. Appl. Phys. Lett. 83(7): 1370-1372

Pramanik C, Islam T, Saha H, Bhattacharya J, Banerjee S, Dey S 2005 Design, fabrication, testing and simulation of porous silicon-based smart MEMS pressure sensor. Proceedings of the $18^{\text {th }}$ International Conference on VLSI design (VLSID-2005) 235-240

Senturia D, Stephen 2001 Microsystem Design, (Boston/Dordrecht/London: Kluwer Academic Publishers) p. 469

Smith R L, Collins S D 1992 Porous silicon formation mechanisms. J. Appl. Phys. 71(8): R1-R22

Stephen Beeby, Graham Ensell, Michael Kraft and Neil White, 2004 MEMS Mechanical Sensors, p. 469, ARTECH House Inc

Sujatha L, Enakshi Bhattacharya 2007 Enhancement in sensitivity of pressure sensors with composite Si/Porous Silicon membrane. J. Micromech. Microeng. 17: p 1605-1610

Tai-Ran-Hsu 2002 MEMS and microsystems design and manufacture, (New York: Tata McGraw-Hill)

Timoshenko P Stephen S, Woinowsky-Krieger, Theory of plates and shells, $2^{\text {nd }}$ edition, (New York: McGraw-Hill) International Editions

Vyatkin, Starkov V, Tzeitlin V, Presting H, Konle J, Konigh U 2002 Random and ordered macropore formation in p-type silicon. J. Electrochem. Soc. 149(1): G70-G76 